\title{
Delay to TKA in Patients Treated with a Multimodal Approach Using High Molecular Weight, Biologically Derived Hyaluronic Acid
}

\author{
Gerard Malanga, ${ }^{a}$ Damion Martins, ${ }^{\mathrm{b}}$ Srinivas Nalmachu, ${ }^{\mathrm{c}}$ Sam Dona, ${ }^{\mathrm{d}}$ Faizan Niazi, ${ }^{\mathrm{e}, *}$ \\ Karina Utkina, ${ }^{e}$ Forough Farrokhyar,${ }^{f, g}$ Akram Alyass,${ }^{f} \&$ Jeffrey Rosen ${ }^{\mathrm{h}}$ \\ aNew Jersey Regenerative Institute, Cedar Knolls, NJ, USA; bAtlantic Health System, Morristown, NJ, USA; 'Mid America \\ PolyClinic, Overland Park, KS, USA; ' $T$ Tri County Orthopedics, Cedar Knolls, NJ, USA; eFerring Pharmaceuticals, \\ Parsippany, NJ, USA; 'Department of Surgery, McMaster University, Hamilton, ON, Canada; ' Department of Health \\ Research Methods, Evidence, and Impact, McMaster University, Hamilton, ON, Canada; 'Department of Orthopaedics \\ and Rehabilitation, New York Presbyterian Queens, Weill Medical College of Cornell University, NY, USA \\ *Address all correspondence to: Faizan Niazi, Ferring Pharmaceuticals, Parsippany, NJ, USA; Tel.: +1-973-796-1600, E-mail: Faizan.Niazi@Ferring.com
}

\begin{abstract}
Background: The primary objective of this study was to determine the effect of single versus multiple rounds of intra-articular hyaluronic acid (IA-HA) in delaying the need for total knee arthroplasty (TKA) in patients with knee OA, and if additional benefits were seen when used in conjunction with other multimodal treatment options.

Methods: This study was a retrospective claims analysis of a large commercial database containing more than 100 million patients with continuous coverage from October 1, 2010 through September 30, 2015. Time to TKA for patients who received one course of Euflexxa (IA-BioHA) were compared to patients who received two or more courses of IA-BioHA and patients who received no IA-HA. Assessment of multimodal treatment effects was done between the following groups: IA-BioHA injections alone, IA-BioHA and bracing, IA-BioHA and corticosteroid injection, and IA-BioHA with both corticosteroids and bracing.
\end{abstract}

Results: A total of 26,727 patients were included in the analysis of treatment courses, and 31,034 in the analysis of multimodal treatment combinations. The use of IA-BioHA demonstrated a delay of TKA that was prolonged with repeated courses of treatment (1.411 years, interquartile range [IQR]: 1.44). The greatest delay to TKA was observed for the patients who had received all three treatment options (1.5 years, IQR: 1.52) in the multimodal analysis.

Conclusions: These results confirm that treatment of knee OA should consider the use of multimodal therapy instead of focusing on individual treatment options. Additionally, the use of repeated courses of IA-BioHA should be considered for prolonged benefit for patients with symptomatic knee OA.

KEY WORDS: knee, osteoarthritis, multimodal, hyaluronic acid, corticosteroid, bracing

\section{INTRODUCTION}

Knee osteoarthritis (OA) is a highly investigated area of health research due to its significant impact on functional disability, stiffness, and pain for patients, a well a large socioeconomic burden for patients and the healthcare system. ${ }^{1,2}$ Patients are typically managed using a variety of nonpharmacologic and pharmacologic treatment options. ${ }^{3,4}$ As the disease progresses to more advanced stages, surgical intervention may be required. In these cases, the knee joint can be treated by performing partial or total knee arthroplasty (TKA). ${ }^{5}$
In patients who have not progressed to the point where they require TKA, or are unwilling to undergo TKA, there is a need for effective treatments to relieve pain and improve function. ${ }^{6}$ It may be advantageous to provide symptomatic relief for these patients, while also delaying TKA at their current stage of disease progression. ${ }^{6}$ There are many nonsurgical treatment options available for the management of knee OA prior to the need for a knee replacement, including NSAIDs, bracing, corticosteroids, and intra-articular hyaluronic acid (IA-HA) injections. Current research typically aims to identify the superiority of these available 
treatment options in comparison to each other, yet a multimodal approach is often utilized by clinicians in the real-world treatment of knee OA. ${ }^{7}$

The use of IA-HA as a treatment option has been shown to be effective in reducing negative outcomes, such as pain, in patients with mild to moderate knee OA. ${ }^{8,9}$ Differences in treatment effect have been demonstrated across the class of IA-HA products, with high molecular weight (HMW) IA-HA products demonstrating greater pain relief than low molecular weight (LMW) products. ${ }^{10} \mathrm{Eu}$ flexxa (IA-BioHA) is one such HMW IA-HA that is biologically derived, forgoing the use of avian-derived molecules that are present in other IA-HA products. ${ }^{11}$ A typical course of IA-BioHA consists of three injections administered over a three-week period. While most IA-HA evidence focuses on the initial course of treatment, some evidence has suggested that the beneficial effects of IA-HA may be prolonged, and even increased, following repeat courses of treatment. ${ }^{12,13}$ These studies demonstrated that repeat courses of IA-BioHA had a dose-response relationship that not only prolonged pain improvement after the initial course but also further increased reductions in pain. ${ }^{12,13}$

The primary objective of this study was to determine the effect of IA-BioHA in delaying the need for TKA in patients with knee OA, both alone, and in multimodal treatment regimens. The analysis looked at the effect of IA-BioHA alone, with one round of injections, repeat rounds of injections, and if there were any additional benefits conferred when used in conjunction with other treatment options through the analysis of real-world evidence.

\section{METHODS}

\section{A. Study Design}

This study was a retrospective claims analysis of a large commercial database containing more than 100 million patients with continuous coverage from October 1, 2010 through September 30, 2015. The database included anonymous claims data for all OA patients seen within the aforementioned timeframe. The database contained HIPAA compliant de-identified data, and as a retrospective assessment of de-identified data, this investigation did not require ethics approval.

\section{B. Eligibility Criteria}

All patients with the diagnosis code for knee osteoarthritis and a treatment received prior to TKA were included. Exclusion criteria were as follows: patients without OA, patients with OA other than knee, patients with knee OA who had immediate TKA after diagnosis and no treatment, and patients with knee OA who had no treatment and no TKA. Patients were excluded if they received any other IA-HA treatment in the 12 months prior to treatment with IA-BioHA to reduce the effects of other IA-HA products on the assessed outcome. Eligibility was defined by ICD- 9 codes. The ICD-9 codes that were included in our analysis of TKA were: ICD-9 711. x6, 712.x6, 715.x6, 716.x6, 717.x, 718.x6, 719.x6, 836.x, and 844.x.

\section{Comparison Groups}

Patients who received one course of IA-BioHA were compared to patients who received two or more courses of IA-BioHA, as well as those who did not receive any IA-HA treatment. Additionally, the assessment of multimodal treatment effects examined the following groups: IA-BioHA injections alone, IA-BioHA and bracing, IA-BioHA and corticosteroid injection, and IA-BioHA with both corticosteroids and bracing. IA-HA is recommended for treatment only after the use of NSAIDs no longer provides therapeutic relief, so most patients were assumed to have typically taken simple analgesics during their OA treatment regimen.

\section{Data Analysis}

Descriptive statistics of included patients were reported as counts and percentages for categorical data. Means and standard deviations were reported for continuous outcomes unless the data were not normally distributed. In this case, the median and interquartile range (IQR) were provided. The delay to TKA for patients who received single and multiple courses of IA-BioHA was assessed from the 
time of the first IA-BioHA injection to provide insight into the delay that may be considered directly attributable to IA-BioHA. Additionally, analysis of delay to TKA was conducted from the time of first OA treatment code, an estimation of the time of diagnosis, for patients who received either one or at least two full courses of IA-BioHA to assess the overall delay period for these patients. Only patients who eventually required TKA were included, and patients who did not require TKA throughout the study period were not included in the analysis. Kaplan-Meier survival curves were provided to illustrate the differences in TKA rates between one course and multiple courses of IA-BioHA throughout the study timeframe. These groups considered all patients who received IA-BioHA, regardless of any other treatment options they may have received. To better understand the direct impacts of specific multimodal therapy regimens on the delay to TKA, a similar analysis was conducted for different multimodal treatment combinations. The assessment of time to TKA in patient subgroups receiving specific multimodal treatments was analyzed from the time of first OA treatment code. Kaplan-Meier survival curves were provided for the following groups: IABioHA injections alone, IA-BioHA and bracing, IABioHA and corticosteroid injection, and IA-BioHA with both corticosteroids and bracing.

\section{RESULTS}

\section{A. Included Patients}

The administrative database included 2,390,375 patients. Among these patients, 1,248,298 patients were diagnosed with knee OA and were over the age of 18 , with no history of prior knee replacement. 563,103 patients did not receive an IA-HA treatment for their knee OA in the 12 months prior to treatment with IA-BioHA. Of these patients, 139,665 eventually had a TKA in the database timeframe (mean age of $56.11 \pm 8.5,57.6 \%$ female). A total of 19,004 patients within the database received one full course of IA-BioHA (mean age $55.5 \pm 8.4,61.7 \%$ female), and 7,723 patients received two or more courses of IA-BioHA (mean age $55.5 \pm 7.9,63.6 \%$ female). There were 10,183 patients who received only IABioHA (mean age $54.8 \pm 8.9,61.2 \%$ female), 699 patients who received IA-BioHA and a prescribed brace (mean age $53.7 \pm 8.8,48.2 \%$ female), 18,422 patients who received both and corticosteroid injection (mean age $55.9 \pm 7.9,64.0 \%$ female), and 1,730 patients who received corticosteroid injection and a prescribed brace (mean age $55.5 \pm 7.9,51.2 \%$ female).

\section{B. Single vs. Multiple Courses of IA-BioHA}

A summary of the number of TKAs and median time to TKA for those who eventually underwent TKA for course groups is provided in Table 1. The median time to TKA from the first injection for the one course group was 0.66 years (IQR: 0.87) and 1.411 years (IQR: 1.44) for the multiple course group. A Kaplan-Meier analysis of the TKA-free survival for treatment course groups is included in Fig. 1; the median time to TKA was 1.36 years (IQR: 1.58 ) for the one IA-BioHA course group and 1.91 years (IQR: 1.66) for the multiple course group. In comparison, the median time to TKA from the first OA treatment code was 0.38 (IQR: 0.95) for patients who received no IA-HA treatment.

TABLE 1: Time to TKA for patients who received single and multiple IA-BioHA courses

\begin{tabular}{|l|c|c|c|c|}
\hline \multicolumn{1}{|c|}{$\begin{array}{c}\text { Treatment } \\
\text { courses }\end{array}$} & $\begin{array}{c}\text { Number of } \\
\text { patients }\end{array}$ & $\begin{array}{c}\text { Number } \\
\text { of TKAs }\end{array}$ & $\begin{array}{c}\text { Median years to TKA from } \\
\text { first IA-BioHA injection } \\
\text { (IQR) }\end{array}$ & $\begin{array}{c}\text { Median years to TKA from } \\
\text { first OA treatment code } \\
\text { (IQR) }\end{array}$ \\
\hline No IA-HA & 563,103 & 139,665 & NA & $0.38(0.95)$ \\
\hline One course & 19,004 & 5,061 & $0.66(0.87)$ & $1.36(1.58)$ \\
\hline $\begin{array}{l}\text { Two or more } \\
\text { courses }\end{array}$ & 7,723 & 1,876 & $1.411(1.436)$ & $1.91(1.66)$ \\
\hline
\end{tabular}




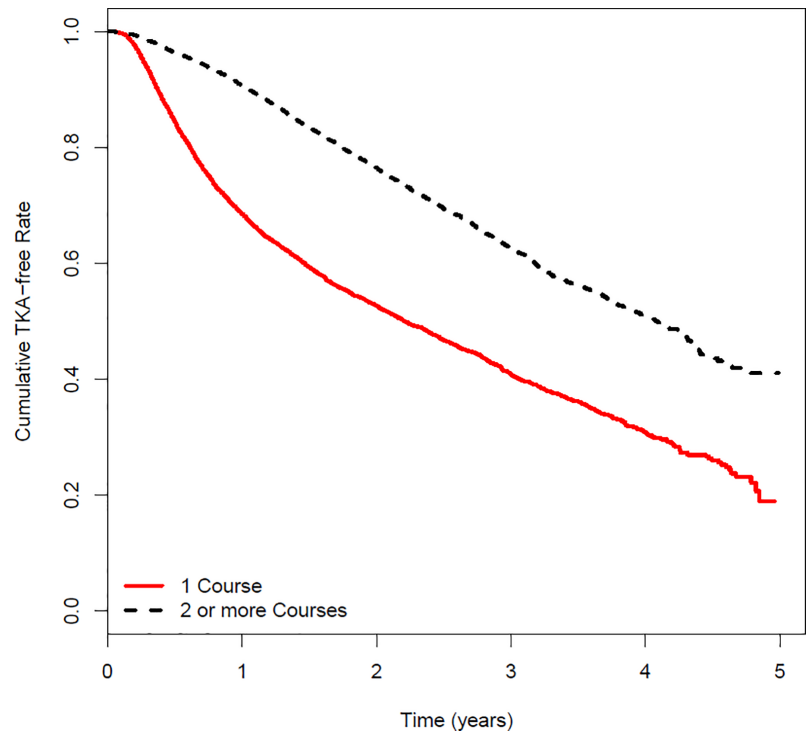

FIG. 1: Kaplan-Meier of treatment courses. Cumulative TKA-free rate over time from first IA BioHA injection in patients who received 1 , or 2 or more courses of IA-BioHA.

\section{Multimodal Therapy}

Table 2 includes a summary of the number of TKAs and the median time to TKA for each of the multimodal treatment groups. Of those that received a TKA, the median time to the event from first OA treatment code was longest for patients who received all three therapies (1.5 years, IQR: 1.52), followed by patients treated with IA-BioHA and corticosteroid (1.32 years, IQR: 1.48), IA-BioHA and a prescribed brace (1.1 years, IQR: 1.43 ), and IA-BioHA alone (0.89 years, IQR: 1.28). A KaplanMeier curve of the TKA-free survival for all groups is shown in Fig. 2.

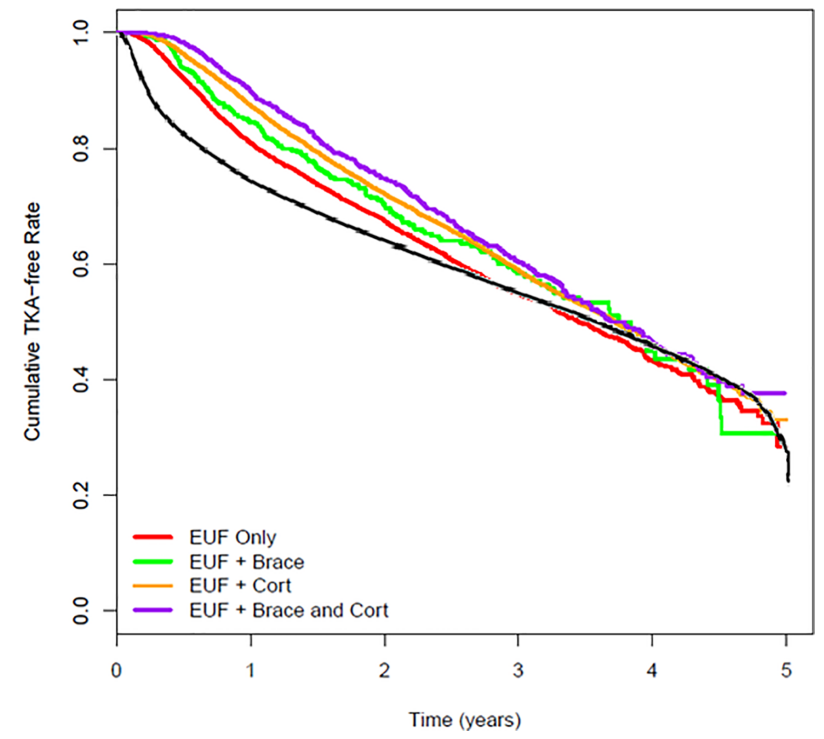

FIG. 2: Kaplan-Meier of multimodal groups. Cumulative TKA-free rate over time from first OA treatment in patients who received no IA-HA, IA-BioHA, IA-BioHA and a brace, IA-BioHA and corticosteroid, or IA-BioHA, a brace, and corticosteroid.

\section{DISCUSSION}

This study identified an additive pattern in which multimodal therapy regimens can prolong the time to TKA. Compared to the use of IA-BioHA alone, the delay to TKA was prolonged with the addition of bracing or corticosteroids, with the greatest benefit seen when all three treatments were used together. This study provides a novel approach to assessing multimodal treatment of knee OA with a large observational database, with findings that confirm those seen within assessments of other multimodal regimens. ${ }^{7}$ This study additionally found that the

TABLE 2: Median time to TKA for multimodal treatment groups

\begin{tabular}{|l|c|c|c|}
\hline \multicolumn{1}{|c|}{ Multimodal groups } & $\begin{array}{c}\text { Number of } \\
\text { Patients }\end{array}$ & $\begin{array}{c}\text { Number of } \\
\text { TKAs }\end{array}$ & $\begin{array}{c}\text { Median years to TKA from first } \\
\text { OA treatment code (IQR) }\end{array}$ \\
\hline No IA-HA & 563,103 & 139,665 & $0.38(0.95)$ \\
\hline IA-BioHA only & 10183 & 2263 & $0.89(1.28)$ \\
\hline IA-BioHA and knee brace & 699 & 180 & $1.10(1.43)$ \\
\hline IA-BioHA and corticosteroid & 18422 & 5026 & $1.32(1.48)$ \\
\hline All three treatments & 1730 & 519 & $1.50(1.52)$ \\
\hline
\end{tabular}


administration of IA-BioHA postponed the need for TKA, and continuing with IA-BioHA treatment beyond the first course may be of greater benefit within the patients who progressed to TKA. Previous investigations have identified the TKA delaying effects of repeated courses of IA-HA injection'; however, this study specifically addresses the differences in effect that are seen after repeated courses of IA-BioHA from real-world evidence. Repeated IA-HA injections have also been shown to increase symptom relief benefits, as pain and function improvements tend to be maintained - if not further improved - by additional courses of IA-HA. ${ }^{12,13}$

The findings of this study raise important considerations for clinical practice, as well as future research. There are two key findings that can be utilized within clinical practice from this study. First, a multimodal approach that utilizes IA-BioHA, corticosteroids, and bracing, can be used to optimize benefits for patients. Clinically, it may also be important to consider the use of IA-HA treatments, such as IABioHA, earlier in the OA disease progression to allow for repeated injection timing to be appropriate for a patient, prior to the patient progressing to a late disease stage. This may not only allow for patients to experience a greater delay until their need for TKA, but also significant and prolonged relief from pain and functional disability associated with knee OA. ${ }^{9}{ }^{14}$ From a research perspective, the findings of this study also have important implications. Currently, the majority of IA-HA evidence focuses on the initial treatment course of individual treatments. There have been far fewer studies that assess multimodal treatment approaches or follow patients for multiple treatment courses, particularly when assessing symptomatic outcomes such as pain and function. ${ }^{13}$ To gain a better understanding of the treatment effects for patients receiving multimodal treatment and repeated courses of treatment, research endeavors should continue to collect patient data beyond their first IA-HA treatment course.

There are important strengths to consider regarding this study. First, the utilization of a large administrative database allows for the analysis real-world data on a scale that is not typically seen in randomized trial settings. This type of data may be more useful to the practicing clinician who will likely experience similar type of patient scenarios versus the more narrowly selected and treated subjects in randomized controlled studies. This current study provides a large enough sample to assess the progression of timing to TKA within patients who received varying multimodal treatment combinations for their knee OA. The differentiation between IA-HA product outcomes has been demonstrated, which makes this assessment of a single treatment valuable in distinguishing brand-specific results from class-specific assessments. ${ }^{10}$

There are also a number of key limitations to consider for this study. While observational data provides the opportunity to assess the treatment pathway and progression to TKA, this study design also poses challenges. First, the data provided within the database was taken at face value by the researchers, as data auditing for accuracy was not possible after the data had been anonymized and sent to the research team. Another important consideration is the inability to assess or determine the candidacy of included patients for TKA. Without specific assessments of disease progression, the requirement of TKA was considered as a sign of disease severity worsening. This may not always be the case, as some patients may face factors other than disease progression that result in them deciding to undergo, or not undergo/be ineligible for, TKA (job status, economics, family needs, etc.). The results of this study assume that patients who receive TKA have progressed to a severe stage of knee OA, which is a reasonable assumption for the majority of knee OA patients. Injection of HA in patients with severe OA is unlikely to be of benefit, based upon prior research, and may result in higher treatment failure. Addressing OA pain in the earlier stages may result in more robust outcomes and a longer delay for the need for TKA. This still poses as a limitation within this study, as direct disease severity data were not collected in the administrative database. Additionally, while the assessment of a multimodal approach suggests that the use of all three treatments provides the greatest benefit, it does not provide specific information regarding the optimal order of use for each of the treatments. Future studies may provide additional clarity on this. Despite these limitations, this project provides a detailed assessment of the 
impact of single and multiple courses of a high molecular weight, biologically derived IA-HA product on patient delay to TKA from a large administrative database.

\section{CONCLUSION}

The results of this study highlight the clinical benefit of IA-BioHA within a multimodal approach to treatment of knee OA. The greatest delay to TKA was observed for patients who received all three treatment options, followed by the groups who received combined therapy of two interventions, and finally the group who received IA-BioHA alone. It is of note that a large proportion of patients were treated with both IA-BioHA and corticosteroids, which demonstrated improved results over IA-BioHA alone and also over IA-BioHA and bracing. The use of IA-BioHA demonstrated a delay in the need for TKA that was prolonged with repeated courses of treatment. These results confirm that treatment of knee OA should consider the use of multimodal therapy instead of focusing on individual treatment options. Additionally, the use of repeated courses of IA-BioHA should be considered for prolonged benefit for patients with symptomatic knee OA. Further research is needed to determine the optimal order and timing of the various multimodal treatments to maximize outcomes.

\section{ACKNOWLEDGMENTS}

This study was funded by Ferring Pharmaceuticals. Medical writing support was provided by Global Research Solutions Inc.

\section{REFERENCES}

1. Rosen J, Avram V, Fierlinger A, Niazi F, Sancheti P, Bedi A. Clinicians' perspectives on the use of intra-articular hyaluronic acid as a treatment for knee osteoarthritis: A North American, Multidisciplinary Survey. Clin Med Insights Arthritis Musculoskelet Disord. 2016;9:21-7.

2. Cross M, Smith E, Hoy D, Nolte S, Ackerman I, Fransen M, Bridgett L, Williams S, Guillemin F, Hill CL, Laslett LL. The global burden of hip and knee osteoarthritis: Estimates from the global burden of disease 2010 study. Ann Rheumatic Dis. 2014 Jul 1;73(7):1323-30.
3. Cooper $\mathrm{C}$, Rannou $\mathrm{F}$, Richette $\mathrm{P}$, Bruyère $\mathrm{O}, \mathrm{Al}$-Daghri $\mathrm{N}$, Altman RD, Brandi ML, Collaud Basset S, Herrero-Beaumont G, Migliore A, Pavelka K. Use of intraarticular hyaluronic acid in the management of knee osteoarthritis in clinical practice. Arthritis Care Res. 2017 Sep;69(9):1287-96.

4. Arnold W, Fullerton DS, Holder S, May CS. Viscosupplementation: Managed care issues for osteoarthritis of the knee. J Manag Care Pharm. 2007 May;13(4 Suppl A):3-23.

5. Losina E, Paltiel AD, Weinstein AM, Yelin E, Hunter DJ, Chen SP, Klara K, Suter LG, Solomon DH, Burbine SA, Walensky RP. Lifetime medical costs of knee osteoarthritis management in the United States: Impact of extending indications for total knee arthroplasty. Arthritis Care Res. $2015 \mathrm{Feb} ; 67(2): 203-15$.

6. Li CS, Pathy R, Adili A, Avram V, Barasi MA, Mundi R, Niroopan G, Bhandari M. Is the treatment gap in knee osteoarthritis real? A qualitative study of surgeons' perceptions. J Long Term Eff Med Implants. 2013;23(2-3):223-40.

7. Miller LE, Block JE. An 8-week multimodal treatment program improves symptoms of knee osteoarthritis: A real-world multicenter experience. Pragmat Obs Res. 2013;4:39-44.

8. Bannuru RR, Schmid CH, Kent DM, Vaysbrot EE, Wong JB, McAlindon TE. Comparative effectiveness of pharmacologic interventions for knee osteoarthritis: A systematic review and network meta-analysis. Ann Intern Med. 2015;162(1):46-54.

9. Dasa V, Lim S, Heeckt P. Real-world evidence for safety and effectiveness of repeated courses of hyaluronic acid injections on the time to knee replacement surgery. Am J Orthop. 2018;47(7).

10. Altman RD, Bedi A, Karlsson J, Sancheti P, Schemitsch E. Product differences in intra-articular hyaluronic acids for osteoarthritis of the knee. Am J Sports Med. 2016;44(8):2158-65.

11. Altman RD, Farrokhyar F, Fierlinger A, Niazi F, Rosen J. Analysis for prognostic factors from a database for the intra-articular hyaluronic acid (Euflexxa) treatment for osteoarthritis of the knee. Cartilage. 2016;7(3):229-37.

12. Altman RD, Rosen JE, Bloch DA, Hatoum HT. Safety and efficacy of retreatment with a bioengineered hyaluronate for painful osteoarthritis of the knee: Results of the open-label extension study of the FLEXX trial. Osteoarthritis Cartilage. 2011;19(10):1169-75.

13. Altman R, Hackel J, Niazi F, Shaw P, Nicholls M. Efficacy and safety of repeated courses of hyaluronic acid injections for knee osteoarthritis: A systematic review. Semin Arthritis Rheum. 2018;48(2):168-75.

14. Nicholls M, Shaw P, Niazi F, Bhandari M, Bedi A. The impact of excluding patients with end-stage knee disease in intra-articular hyaluronic acid trials: A systematic review and meta-analysis. Adv Ther. 2019;36(1):147-61. 